\title{
Unravelling the immunotoxicity of polycaprolactone nanoparticles - effects of polymer molecular weight, hydrolysis and blends
}

Sandra Jesus ${ }^{\dagger}$, Natalia Bernardi $\ddagger$, Jessica da Silva ${ }^{\ddagger}$, Mariana Colaço ${ }^{\dagger, \ddagger}$, João Panão Costa ${ }^{\dagger, *}$, Pedro Fonte $\$, \|, \perp$ and Olga Borges $* \dagger, \star$

$\dagger$ Center for Neuroscience and Cell Biology, University of Coimbra, 3004-504, Coimbra, Portugal

$\$$ Faculty of Pharmacy, University of Coimbra, 3000-548, Coimbra, Portugal

$\S$ Center for Marine Sciences (CCMAR), University of Algarve, Gambelas Campus, 8005-139 Faro, Portugal

\| Department of Chemistry and Pharmacy, Faculty of Sciences and Technology, University of Algarve, Gambelas Campus, 8005-139 Faro, Portugal

${ }^{\perp} i B B$ - Institute for Bioengineering and Biosciences, Department of Bioengineering, Instituto Superior Técnico, Universidade de Lisboa, 1049-001 Lisboa, Portugal 

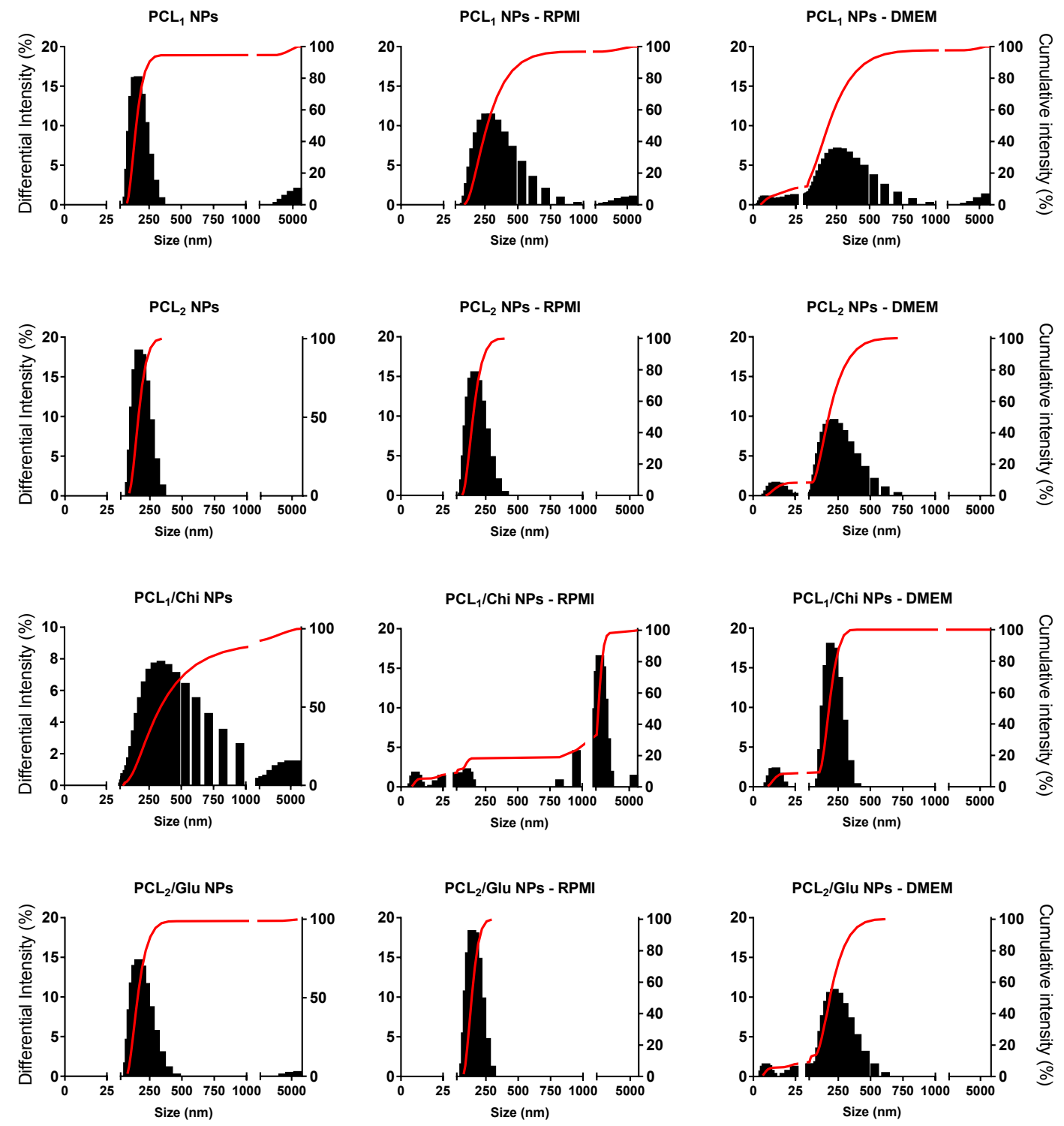

Figure S1: Size distribution of the different PCL based NPs measured by DLS in water and $24 \mathrm{~h}$ after resuspension in the cell culture media (RPMI and DMEM). Each graphic is representative replicate of a measurement selected from the 3 independent measurements performed for each condition. 